\title{
REVERSE-LINK POWER ALLOCATION IN TWO-HOP MULTIMEDIA CDMA NETWORKS
}

\author{
Dave Walsh and Halim Yanikomeroglu \\ Broadband Communications and Wireless Systems (BCWS) Centre \\ Dept. of Systems and Computer Engineering, Carleton University, Canada \\ $\{$ dwalsh,halim\}@sce.carleton.ca
}

\begin{abstract}
Recently there has been significant interest in augmenting the cellular networks with the multihop capability to obtain better high data rate performance in the context of enhanced-3G and beyond-3G networks.

This paper deals with the power allocation strategies in the reverse-link of two-hop multimedia CDMA networks. In this envisioned network, the WTs (wireless terminals) which cannot establish a direct link with the BS (at the required rates) seek the assistance of those WTs which can; in other words, whenever needed, some WTs are used as relayers for some other WTs (relayees) if this is possible. In a two-hop link, the first hop (relayee to relayer) uses the unlicensed band and the second hop (relayer to BS) uses the cellular band. This arrangement not only guarantees that no additional expensive cellular spectrum will be used to facilitate a two-hop link, but it also guarantees that if anything goes wrong in the first hop, this will not affect the performance of the WTs which directly communicate with the BS in the cellular band.

The performance of any CDMA network depends on the implementation of a good power allocation and control scheme. The optimum power allocation and control in the reverse link of the conventional single-hop CDMA networks is well known. The main contribution of this paper is the development of a good power allocation scheme (which is tied to the proper selection of relayers) in the first hop of the two-hop reverse-link of the envisioned CDMA network. It is worth emphasizing that the nature of the reverse-link power allocation problem is very different in the many one-to-one links (or possibly numerous several-to-one links) which collectively constitute the first hop of the two-hop CDMA network under consideration, in comparison to that in the single many-to-one link in the reverse link of a conventional single-hop CDMA network.

The simulation results show that the two-hop relaying, facilitated with the developed novel power allocation scheme, yields considerable enhancements in the CDMA cell capacity, coverage, and throughput.
\end{abstract}

Keywords: multihop CDMA; power allocation; relay

\section{INTRODUCTION}

Relaying is considered for future wireless networks because it can significantly enhance the high data rate coverage and can increase the throughput in beyond-3G networks [1]. We consider the reverse-link of two-hop multimedia CDMA networks in this paper.
Similar to any other CDMA network, multihop CDMA networks are also interference limited, rendering effective power allocation essential. Two different frequency bands are assumed to be used in the two-hop network in order to decouple the two hops: the license-exempt band in the first hop (relayee to relayer) and the cellular band in the second hop (relayer to the BS). The second hop of the two-hop CDMA network is in effect identical to the reverse-link of a conventional single-hop CDMA network; in both cases there exist a single many-to-one link (from interference management point of view), for which the power allocation is well known [2].

However, power allocation in the first hop of a two-hop CDMA network is a new problem which has never been addressed in the very rich power control literature as far as we know. This is due to the fact that the first hop in a two-hop network is composed of many one-to-one (or possibly numerous several-to-one) links; this new topology emerges only in the context of multihop communications.

Our initial research indicates that finding an optimal power allocation scheme (which is tied to optimal relayer selection) is not an easy problem [3]. It is not clear to us whether various power allocation schemes developed in the literature can readily be used in this different topology.

We have developed a power allocation algorithm for the above described two-hop multimedia CDMA network. Although we do not know how far away our algorithm is from the optimum power allocation, simulation results indicate that the developed algorithm works well. It is the development of a power allocation algorithm for this new topology (many one-to-one or several-to-one links) which distinguishes this paper from some other recent publications on CDMA relaying networks, such as $[4,5]$.

In addition to this novel power allocation algorithm, a relayer selection scheme which incorporates fair resource allotment and avoids bottlenecks is also developed during this project [3]; however, due to space constraints it is not described in this paper.

In our system, some WTs may not be able to connect to the BS in single-hop due to inadequate SINR (signal-to-interferenceplus-noise ratio); however, the WTs that are connected to the BS, candidate relayers, may be used to relay the signals of the unconnected WTs (relayees) to the BS. It should be noted that 
candidate relayers become relayers when they transmit signals received from relayees to the BS.

In this project relaying is done in the unlicensed bands $(5 \mathrm{GHz})$ and the connection to the BS occurs in the licensed bands (2 $\mathrm{GHz}$ ). In other words, the first hop takes place in the unlicensed bands while the second hop takes place in the licensed bands; therefore, each WT will need two air interfaces, one at $2 \mathrm{GHz}$ and another at $5 \mathrm{GHz}$. The unlicensed bands are used for relaying because they are free, so the performance of the network can be enhanced without the cost of additional spectra license fees. In addition, by using the unlicensed bands, power control errors during relaying and the transmissions of the relayees will not affect the WTs in the licensed bands.

The system used in this paper consists of a single hexagonal cell with no mobility. However, the system can be extended to include a multi-cell environment. Shadow fading is used in both hops (standard deviation $=8 \mathrm{~dB}$ ); however, multipath fading is excluded. Multipath fading is excluded because of its fast changing nature (in both time and distance). These rapid changes are likely to result in relayer selection oscillation, in which a relayee switches between relayers due to the fast changing links. CDMA is used in both the licensed and unlicensed bands and the WTs are placed in a hexagonal cell with a centrally located BS; the CDMA schemes used will be described later.

\section{ALGORITHM DEVELOPMENTS}

\subsection{Power Allocation: Single-Hop Case}

An optimal power allocation scheme for single hop CDMA networks was previously derived in [2]. In this scheme, the receive power that should be allocated to each WT at the BS in order to realize adequate service is:

$$
P_{i}=\frac{f_{i} P_{N}}{1-\sum_{j=1}^{K} f_{j}}
$$

where

$P_{i}=$ minimum receive power necessary at the BS for WT $i$ to get adequate service,

$f_{i}=$ fractional consumption of WT $i$,

$P_{N}=$ noise power, and

$K=$ number of WTs in the system.

The $\sum_{j=1}^{K} f_{j}$ term in (1) includes $f_{i}$ and is the sum of the fractional consumptions of all the WTs in the system. Please note that the term "fractional consumption" is a new term [2]; it is a counting mechanism to keep track of a system's capacity consumption.

In [6] the term $f_{i}$ is expressed as

$$
f_{i}=\frac{\gamma_{i}}{N_{(i)}+\gamma_{i}}=\frac{R_{i} \gamma_{i}}{W+R_{i} \gamma_{i}}
$$

where
$W=$ system bandwidth,

$N_{(i)}=W / R_{i}=$ spreading gain for WT $i$,

$R_{i}=$ bit rate for WT $i$, and

$\gamma_{i}=$ required SIR for WT $i$.

The fractional consumption of each WT in the system limits the number of WTs that can be accommodated, so from [3] we now have

$$
\sum_{l=1}^{L} K_{l} f_{l}<1
$$

where $L$ is number of different WT classes, and $K_{l}$ is the number of WTs in class $l$.

Equation (3) indicates that more resources cannot be consumed beyond what is available. Also, each class mentioned above consists of a group of WTs which have the same bit rate, SIR and spreading gain. Please note that (3) never allows $100 \%$ of the system's resources to be used; therefore, the denominator in (1) never equals zero.

\subsection{Power Allocation: Two-Hop Case}

Neither the power allocation scheme shown in section 2.1 nor the system developed in [7] can be used in a two hop system because the first hop is decentralized (i.e. there is no one recipient of all the signals that will ensure that they all meet their minimum targets or be cut off) and both Section 2.2 and [7] assume centralized systems. Implementation of (1) demands centralization because the equation assumes that all the WTs' signals received at the BS meet their target values, and that those that do not are cut off. However, this is not the case for hop 1. On hop 1 there may be several WT signal recipients acting as mini base stations, each employing power control to ensure that the relayees using it achieve their targets. In this scenario, there may be significant interference from the relayees using adjacent relayers. The received powers from these interfering signals undermines the assumptions in (1), rendering it unusable for hop 1. It should be noted that each relayer may see two different types of interference: one from the WTs that are using it (these would be received at their target values) and the other from WTs that are using other relayers (these would be received at levels other than their target values). It is the latter type of interference that undermines the assumptions inherent in (1). It should be emphasized that the following power allocation algorithm development is the main analytical contribution of this paper and is a first in the literature:

$$
\gamma_{(i, k)}=N_{(i)} \frac{P_{R(i, k)}}{\sum_{\substack{j=1 \\ j \neq i}}^{K_{R}} P_{R(j, k)}+P_{N}}=\frac{N_{(i)} P_{T(i)} G_{(i, k)}}{\sum_{\substack{j=1 \\ j \neq i}}^{K_{R}} P_{T(j)} G_{(j, k)}+P_{N}}
$$

where

$\gamma_{(i, k)}=$ SINR of WT $i$ at relayer $k$,

$P_{R(i, k)}=$ receive power of WT $i$ at relayer $k$,

$P_{R(j, k)}=$ receive power of interferer $j$ at relayer $k$,

$K_{R} \quad=$ number of relayees,

$G_{(i, k)}=$ link gain between relayee $i$ and relayer $k$,

$G_{(i, k)}=$ link gain between interferer $j$ and relayer $k$, 
$P_{T(i)}=$ actual transmit power of WT $i$,

$P_{T(j)} \quad=$ actual transmit power of interferer $j$, and

$P_{N}=$ noise power.

Equation (4) can be modified using the following assumptions:

- The WT of interest (WT $i$ ) is transmitting at its maximum power, $P_{T, \max (i)}$.

- $\quad$ The minimum SINR is maintained at the receiver (i.e. the relayer).

- Under the above conditions the transmission link cannot be below a certain minimum (providing the interference does not change), or else the relayee's target SINR value cannot be met.

Using the above assumptions, (4) becomes:

$$
\gamma_{\min (i, k)}=\frac{N_{(i)} P_{T, \max (i)} G_{\min (i, k)}^{\text {hop } 1}}{\sum_{\substack{j=0 \\ j \neq i}}^{K_{R}-1} P_{T(j)} G_{(j, k)}+P_{N}}
$$

After rearrangement we get:

$$
G_{\min (i, k)}^{h o p 1}=\frac{\gamma_{\min (i, k)}\left(\sum_{\substack{j=0 \\ j \neq i}}^{K_{R}-1} P_{T(j)} G_{(j, k)}+P_{N}\right)}{N_{(i)} P_{T, \max (i)}},
$$

where

$G_{\min (i, k)}^{h o p}=$ minimum acceptable hop 1 link gain between WT $i$ and relayer $k$,

$\gamma_{\min (i, k)}=$ minimum acceptable SINR of WT $i$ at relayer $k$ (obtained from specifications), and

$P_{T, \max (i)}=$ maximum transmit power for WT $i$ (not necessarily its actual transmit power).

It should be noted that the specifications are the parameters that must be met for the WT to obtain adequate service. The specifications include the bit rate, the minimum SINR and the spreading gain.

The reasons for the preceding assumptions should be more apparent after inspecting (4), as it can now be seen that $G_{\min (i, k)}^{h o p 1}$ can only be a minimum value if $\gamma_{(i, k)}$ and $P_{T(j)}$ are at their minimum and maximum values respectively. All the other variables in (4) cannot be varied (i.e., $P_{N}$ cannot be changed, $P_{T(j)}$ and $G_{(j, k)}$ must reflect the actual transmit powers and link gains for the interferers, and $N_{i}$ is fixed from the specifications) so only $\gamma_{(i, k)}$ and $P_{T(j)}$ can be manipulated. Since the relayees' transmit powers are changing until the system reaches a state of equilibrium, (5) has to be solved iteratively. In addition:

$P_{R, \min (i, k)}^{\text {hop } 1}=G_{\min (i, k)}^{\text {hop } 1} P_{T, \max (i)}$, and
$P_{R, \min (i)}^{h o p 1}=$ minimum receive power for relayee $i$ at relayer $k$ on hop1.

\section{SIMULATION RESULTS}

The simulation model and results are discussed in this section. In the model, a close in reference distance (i.e. $d_{0}$ ) of 10 meters is used. Also, since an urban environment is being simulated, a propagation constant of 4.0 is employed. A noise power of 1.306e-13 Watt was selected based on the following criteria: a 5 $\mathrm{MHz}$ bandwidth, a $300 \mathrm{~K}$ temperature and an $8 \mathrm{~dB}$ noise figure at the receiver.

The simulation results consist of throughput curves which show the average throughput of the system as the number of WTs (consisting of a combination of class A and class B WTs) increase, left to right, from 1 to 35 . Each throughput figure consists of the following three curves:

- The 'Performance Benchmark' curve has no power cap (i.e. the maximum transmit power) but has resource restrictions and is the benchmark by which the system's performance is judged.

- The 'Without Relaying' curve shows the results obtained when there is no relaying and there are power and resource restrictions.

- The 'With Relaying' curve is the 'Without Relaying' curve with relaying added.

The simulation parameters and the symbols used in Figures 1 and 2 are shown below in Table 1.

\section{Table 1: Parameters and symbols}

$\mathrm{Ra}$ (class A bit rate) $=10 \mathrm{Kbits} / \mathrm{sec}$
$\mathrm{Rb}$ (class $\mathrm{B}$ bit rate) $=50 \mathrm{Kbits} / \mathrm{sec}$

$S I N R a=S I N R b=10$ (classes A \& B SINR respectively)

Radius=1732 $\mathbf{m} \rightarrow \mathbf{A 1} \mathbf{x}, \mathbf{y}$ : without relaying, A2x,y: with relaying

Radius $=\mathbf{1 0 0 0} \mathbf{m} \rightarrow \mathbf{B} 1 \mathbf{x}, \mathbf{y}$ : without relaying, $\mathbf{B 2 x}, \mathbf{y}$ : with relaying

Radius $=\mathbf{5 0 0} \mathbf{m} \rightarrow \mathbf{C 1 x}, \mathbf{y}$ : without relaying, $\mathbf{C 2 x}, \mathbf{y}$ : with relaying

Performance Benchmark $\rightarrow$ D

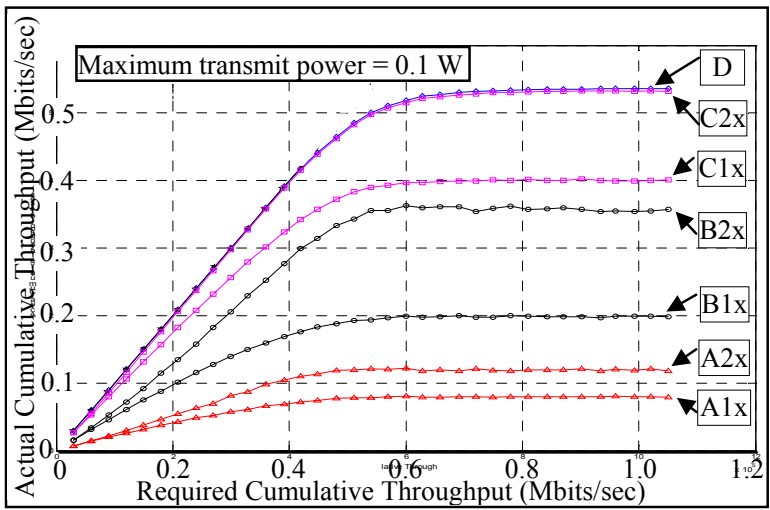

Figure 1: Throughput curves for $P_{\max }=0.1 \mathrm{~W}$

It is apparent from Figure 1 that relaying yields significant gains in small cells when the maximum transmit power is low. This can be seen by comparing curves $\mathrm{C} 1 \mathrm{x}$ and $\mathrm{C} 2 \mathrm{x}$ in Figure 1. Medium size cells also experience significant relaying gains 
under the same conditions; a comparison of curves $\mathrm{B} 1 \mathrm{x}$ and $\mathrm{B} 2 \mathrm{x}$ in Figure 1 will confirm this. However, large cells do not benefit significantly from relaying primarily due to poor link gains, this is evident from comparing curves $\mathrm{A} 1 \mathrm{x}$ and $\mathrm{A} 2 \mathrm{x}$ in Figure 1.

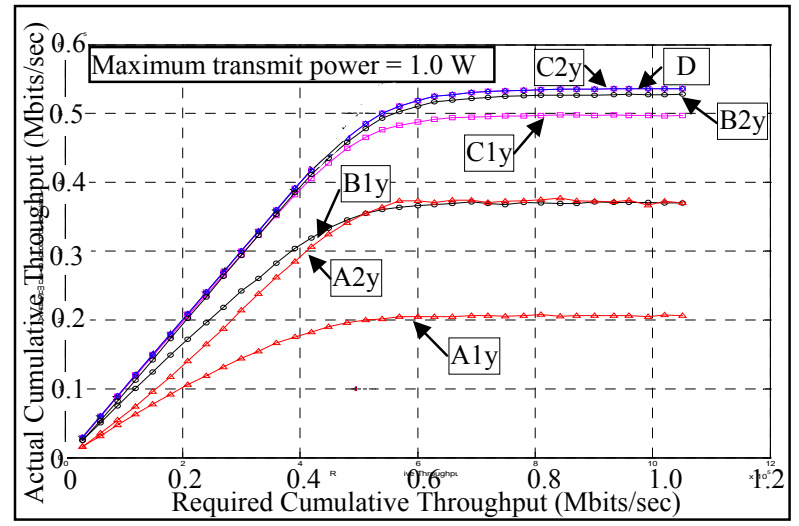

Figure 2: Throughput curves for $P_{\max }=1.0 \mathrm{~W}$

When the power cap is high (i.e., $1.0 \mathrm{~W}$ ) relaying does not yield significant gains in small cells because a large portion of the links between the WTs and the BS are good. This can be borne out by comparing curves $\mathrm{C} 1 \mathrm{y}$ and $\mathrm{C} 2 \mathrm{y}$ in Figure 2. Therefore, in small cells, low power caps are necessary in order to obtain significant throughput increases. Medium and large size cells experience substantial throughput increases when relaying is employed, as is evident from examining curves A1y, A2y, B1y and B2y. However, another very important result can be derived from Figure 2, and that is the potential of this relaying scheme to enable range extension (i.e. the use of fewer BSs to cover a given area). Range extension can result in substantial cost savings. This can be confirmed by comparing curves $\mathrm{A} 2 \mathrm{y}$ and B1y in Figure 2, where one $\mathrm{BS}$ is able to provide the coverage of three BSs $\left([1732 / 1000]^{2}=3\right)$. Therefore, the performance attainable with a $1000 \mathrm{~m}$ radius cell can be obtained in a $1732 \mathrm{~m}$ cell without having to build additional BSs.

\section{CONCLUSIONS}

The two-hop relaying scheme presented in this paper yields significant throughput gains for small cells (i.e. cells with a radius of approximately $500 \mathrm{~m}$ ) only when the power cap is low. However, medium size cells (i.e. cells with a radius of approximately $1000 \mathrm{~m}$ ) experience substantial throughput gains with high and low power caps, while large cells (i.e. cells with a radius of approximately $1700 \mathrm{~m}$ ) only yield significant gains with this relaying scheme when the power cap is high. Also, the relaying scheme presented here not only improves the throughput of multimedia CDMA networks, it also enhances their coverage and capacity. The coverage is increased by extending the system's range to include more distant WTs and the capacity is increased by adding at least some of the unconnected WTs to the system.

The range extension resulting from the implementation of the relaying scheme described in this paper is significant. This outcome is highly desirable because it can result in considerable monetary savings, as one BS employing relaying may be able to provide the same level of service as a much smaller cell which does not utilize relaying. Therefore, it may not be necessary to build additional BSs to give sufficient service to a larger area.

\section{Acknowledgements}

We express our gratitude to CITO for funding this project.

\section{References}

[1] Y.-D. Lin and Y.-C. Hsu, "Multihop cellular: a new architecture for wireless communications", IEEE INFOCOM 2000, vol. 3, pp. 1273-1282, March 2000.

[2] L.C. Yun and D.G. Messerschmitt, "Power control for variable QOS on a CDMA channel", IEEE Military Communications Conference (MILCOM'94), vol. 1, pp. 178182, Oct. 1994.

[3] D. Walsh, Two-Hop Relaying in CDMA Networks Using the Unlicensed Bands, M.A.Sc. thesis (supervisor $\mathrm{H}$. Yanikomeroglu), Carleton University, January 2004.

[4] K. Yamamoto and S. Yoshida, "Call admission control suitable for two-hop CDMA cellular systems", IEEE Vehicular Technol. Conf. Fall 2003 (VTC'F03), Oct. 2003.

[5] A. Fujiwara, S. Takeda, H. Yoshino, and T. Otsu, "Area coverage and capacity enhancement by multihop connection of CDMA cellular network," IEEE Vehicular Technology Conf. Fall 2002 (VTC'F02), pp. 2371- 2374, Sept. 2002.

[6] H. Yanikomeroglu, B. Hashem, and M.H. Ahmed, "Radio resource management (RRM) in wireless multimedia networks", tutorial notes, IEEE Globecom 2002, Taipei, Taiwan, Nov. 17, 2002.

[7] A. Sampath, P.S. Kumar, and J.M. Holtzman, "Power control and resource management for a multimedia CDMA wireless system", IEEE Int.'l Sym. on Personal, Indoor and Mobile Radio Commun. (PIMRC'95), vol. 1, pp. 21-25, Sept. 1995. 